\title{
Dynamics of Erythroid Progenitors and Erythroleukemia
}

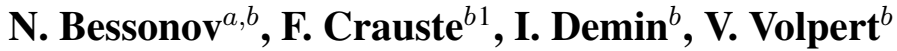 \\ ${ }^{a}$ Institute of Problems of Mechanical Engineering, St. Petersburg, 199178 Russia \\ ${ }^{b}$ Université de Lyon, Université Lyon 1, CNRS, UMR 5208, Institut Camille Jordan, \\ Batiment du Doyen Jean Braconnier, 43, blvd du 11 novembre 1918, \\ F - 69222 Villeurbanne Cedex, France
}

\begin{abstract}
The paper is devoted to mathematical modelling of erythropoiesis, production of red blood cells in the bone marrow. We discuss intra-cellular regulatory networks which determine self-renewal and differentiation of erythroid progenitors. In the case of excessive self-renewal, immature cells can fill the bone marrow resulting in the development of leukemia. We introduce a parameter characterizing the strength of mutation. Depending on its value, leukemia will or will not develop. The simplest model of treatment of acute myeloid leukemia with chemotherapy allows us to determine the conditions of successful treatment or of its failure. We show that insufficient treatment can worsen the situation. In some cases curing may not be possible even without resistance to treatment. Modelling presented in this work is based on ordinary differential equations, reaction-diffusion systems and individual based approach.
\end{abstract}

Key words: erythropoiesis, leukemia, reaction-diffusion systems, cellular modelling AMS subject classification: $35 \mathrm{~K} 57,92 \mathrm{C} 37$

\section{Introduction to erythropoiesis}

Erythropoiesis is the process of production and regulation of red blood cells. It is part of a more general process, hematopoiesis, which allows the formation of all blood cells: white cells, platelets, and red blood cells. All blood cells originate from pluripotent stem cells, called hematopoietic stem cells, which have unique ability to differentiate in one of the three blood lineages $[12,43]$.

\footnotetext{
${ }^{1}$ Corresponding author. E-mail: crauste@math.univ-lyon1.fr
} 
Hematopoiesis occurs in the bone marrow where cells proliferate, differentiate, and ultimately mature cells enter the bloodstream.

During erythropoiesis, cells undergo several divisions, with immature (stem cells, progenitors) and mature stages (reticulocytes, erythrocytes), to finally release cells that will be devoted to oxygen transport in blood. Erythropoiesis is based on a balance between erythroid cell self-renewal (the ability for a mother cell to produce daughter cells with the same maturity level [42]), differentiation (the production of daughter cells more mature than the mother cell) and apoptosis (programmed cell death [37]). Self-renewal is usually considered to be a property of hematopoietic stem cells only, yet recent studies [21,30] showed that erythroid progenitors can self-renew, especially in stress situations. In erythropoiesis, mature cells almost only differentiate, so only progenitors (immature cells) are concerned with self-renewal, differentiation and apoptosis.

These three processes are regulated by numerous intracellular proteins and growth factors. Among all growth factors, glucocorticoids (lipophilic hormones) and erythropoietin (glucoprotein) play particular roles. Glucocorticoids have been shown to regulate erythroid progenitor selfrenewal in stress erythropoiesis [7, 34]. Erythropoietin has been proved to act at various stages in erythroid progenitor maturation, and especially to inhibit erythroid progenitor apoptosis [23]. Among the proteins regulating erythroid cell fate, some key proteins have already been identified. We can cite c-Kit, the protein associated with the stem cell factor (SCF) [28], proteins from the JAK family [38], etc. Recently, Rubiolo et al. [34] identified two proteins, Erk and Fas, as key proteins involved in two antagonist loops acting for erythroid progenitor self-renewal and differentiation/apoptosis, respectively.

Mathematical modelling of hematopoietic cell dynamics has attracted much attention since the end of the 1970's. A pioneering work was proposed by Mackey [26] in 1978. This model describes hematopoietic stem cell dynamics using a system of delay differential equations. The approach proposed in [26] has been the basis for many works devoted to the description of oscillatory behaviors within hematopoietic systems. For instance, in [9] Bernard et al. focused on the white blood cell production to bring an explanation to oscillatory behaviors observed in patients with cyclical neutropenia. See also Adimy et al. [4]. In [32] Pujo-Menjouet and Mackey used a similar model to investigate the appearance of oscillations in blood cell count within patients with chronic myelogenous leukemia. Causes of periodic chronic myelogenous leukemia were also investigated by Adimy et al. [2,3]. A global model of hematopoietic cell dynamics has been proposed by Colijn and Mackey [14, 15] in 2005, and has been applied to the study of cyclical neutropenia and periodic chronic myelogenous leukemia. Recently, models incorporating the action of growth factors on hematopoietic cell dynamics have been considered, see for instance Adimy et al. [1,5], and the references therein. Mathematical models of hematopoiesis applied to neutropenia have also been recently proposed by Vainstein et al. [39, 40], using pharmacodynamics-pharmacokinetics approaches. Mechanisms of leukemias have been investigated by Panetta et al. [31], Shoshat et al. [36], and the references therein.

In 1995, Bélair et al. [8] considered a model of erythropoiesis with a feedback on stem cells from red blood cells mediated by erythropoietin. In [1], Adimy and Crauste enriched the Mackey's model [26] by adjusting erythropoietin effect on the apoptosis rate of erythroid progenitors, in agreement with Koury and Bondurant [23]. More recently, Crauste et al. [16] and Demin et 
al. [19] proposed new models of erythropoiesis focusing on progenitor dynamics and taking into account self-renewal ability of erythroid progenitors, contrary to what had been done in all the above mentioned works.

In this paper, we investigate erythroid cell dynamics by means of a reaction-diffusion system describing cell dynamics and a system of ordinary differential equations describing concentrations of intra-cellular proteins involved in the regulation of self-renewal, differentiation and apoptosis. This model is used to focus on erythroleukemia, a cancer of red blood cells characterized by a rapid increase of immature and abnormal erythroid progenitors in the bone marrow and the blood. Erythroleukemia, or Di Guglielmo syndrome [27], represents in the United States about 5\% of de novo acute myeloid leukemias, and between $20 \%$ and $30 \%$ of secondary leukemias.

Spatial cell distribution can play an important role in hematopoietic cell dynamics because mature cells (reticulocytes) increase differentiation of immature cells when they are close to each other [18]. Moreover, cells push each other in the bone marrow creating pressure difference. It determines their motion and sorting out to blood vessels. Finally, when cell concentration becomes sufficiently high, they can exchange signals which decrease their proliferation and increase apoptosis. Spatial models of hematopoiesis have been previously used, in particular by Ducrot and Volpert [20] to model the development of myelogenous leukemia.

The modelling of the intra-cellular regulatory network is similar to the one described in Roeder and Glauche [33], where the authors considered the interaction between two transcription factors, GATA-1 and PU.1, involved in lineage commitment, and concluded the existence of bistability in their model, i.e. the existence of two stable fixed points, each one corresponding to promotion of one of both lineages. It has also been studied by Huang et al. [22]. Demin et al. [19] proposed a similar model for intra-cellular erythropoiesis regulation.

We consider that erythroid progenitors have the ability to self-renew. This assumption has been used recently by Crauste et al. [16]. Authors concluded that adding self-renewing capacity to erythroid progenitors allows better reproduction of experimental data describing stress erythropoiesis (severe anemia). Several erythroid progenitor maturation levels, depending on the maturity, are considered. Two proteins, Erk and Fas, are assumed to be determining for choice between selfrenewal, differentiation and apoptosis of erythroid progenitors [34]. The interaction between them is supposed to have a bistable behavior [19, 33].

The intra-cellular regulatory network is described and analyzed in Section 2. It is later used, in Section 4, in order to determine the rates of self-renewal and differentiation of erythroid progenitors. Mechanisms of cell motion are discussed in Section 3 and modelled with the individual based approach (cell dynamics software [10]). We show the existence of random motion due to cell division and of directed motion due to cell pressure. The contribution of each of these two factors can be different. In particular, if the cell density is low, then their random motion prevails over directed motion. In this case we can use reaction-diffusion systems of equations in order to describe the evolution of cell populations.

In Section 4 we model normal and leukemic erythropoiesis with reaction-diffusion systems. This analysis allows us to introduce the notion of strength of mutation. If this parameter exceeds a critical value then leukemia will develop. If the mutation is not strong enough, then the influx of normal cells from the stem cell compartment will not allow leukemia to develop. It is interesting 
to note that suppression of leukemia by the influx of normal cells has already been observed, though with another mechanism: in $[10,20]$ it is related to directed (convective) cell motion. A simple model of leukemia treatment with chemotherapy is proposed and shows that treatment can obviously lead to curing, yet in some cases an inappropriate drug dose can worsen the situation.

\section{Intracellular regulation network}

Rubiolo et al. [34] recently investigated regulatory mechanisms of erythroid differentiation. They identified key proteins involved in erythroid progenitor self-renewal and those involved in erythroid progenitor differentiation and apoptosis (see Figure 1). For each cell fate, self-renewal and differentiation/apoptosis, they gave a regulatory pathway made of three proteins: Raf-1, Mek and Erk for the self-renewal loop, Fas, ASK-1 and JNK/p38 for differentiation/apoptosis. A source term for Raf-1 activation appears in their scheme, glucocorticoids (lipophilic hormones), however other source terms do not appear in this scheme and should be taken into account: erythropoietin (glucoprotein) is known to activate erythroid progenitor proliferation and inhibit their apoptosis [23], Fas-ligand (membrane protein) activates Fas [18], for instance.

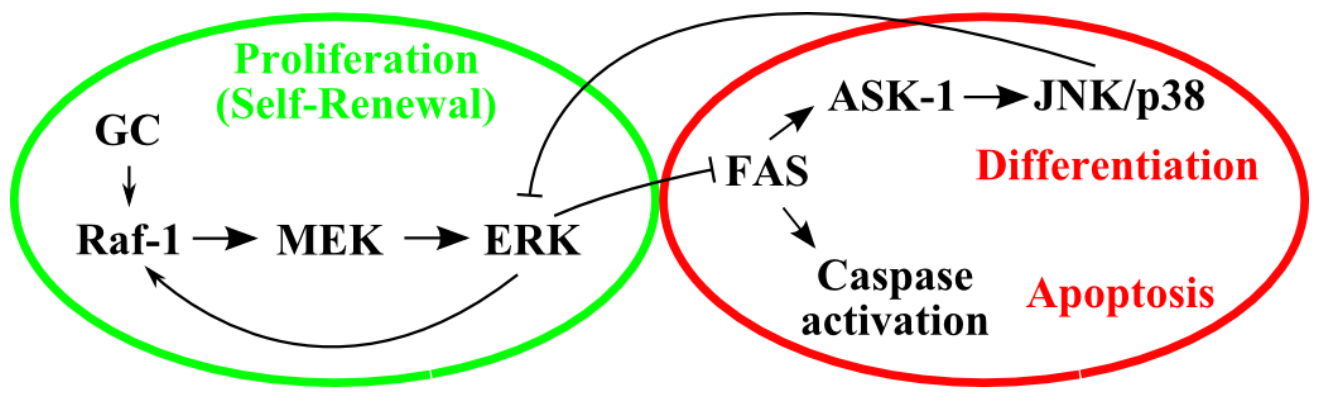

Figure 1: Intracellular regulatory network, adapted from [34]. Two self-sustaining loops competing with each other are presented. Activation of Raf-1 - MEK - Erk reaction cascade induces erythroid cell self-renewal, whereas Fas - ASK1 - JNK/p38 cascade initiates cell differentiation which in presence of low erythropoietin levels activates caspase cascade that results in cell apoptosis.

Two proteins appear of particular importance in this process: Erk and Fas. The former is part of an auto-activating self-renewing loop and inhibits the differentiation/apoptosis regulatory pathway, the latter induces differentiation and apoptosis and inhibits Erk. We focus on these two proteins and describe their interactions by means of a system of ordinary differential equations.

Denote by $\alpha$ and $\gamma$ the external sources of Erk and Fas activator, respectively. For instance, $\alpha$ stands for the activation by glucocorticoids and erythropoietin, and $\gamma$ by Fas-ligand. Let $\beta$ denotes the rate of Erk self-activation. This self-activation is supposed to be nonlinear, with a sensitivity $k \geq 1$.

Due to limited resources, we assume there is a maximal value that limits the production of Erk, denoted by $E_{0}$. One may notice that a maximal value limiting the production of Fas could also be 
introduced, yet thanks to the structure of the second equation in (2.1) a simple change of notations would enable to get a similar equation. Hence such an assumption is not used.

Let denote by $a$ and $d$ respectively Erk and Fas degradation rates. Also denote by $b$ the rate of Erk expression suppression, due to the action of Fas (see Figure 1). This rate then depends on Fas concentration, denoted by $F$, and this dependence is supposed to be linear. Moreover, in agreement with mass action law, we assume $b$ is also proportional to the concentration of Erk, denoted by $E$. The Erk-dependent suppression rate of Fas expression is denoted by $c$.

Then we obtain the following system that describes a simplified version of the regulatory network presented in Figure 1 based on Erk and Fas concentrations,

$$
\begin{cases}\frac{d E}{d t}=\left(\alpha+\beta E^{k}\right)\left(E_{0}-E\right)-a E-b E F & :=\Phi(\alpha, E, F), \\ \frac{d F}{d t}=\gamma-c E-d F & :=\Psi(\gamma, E, F),\end{cases}
$$

where coefficients $a, b, c$ and $d$ are positive.

In the following, we assume $\alpha>0$ and $\gamma>0$, and we focus on the existence of stationary points of (2.1).

The zero lines of the right-hand sides of these equations are given by the formula

$$
F=\frac{\left(\alpha+\beta E^{k}\right)\left(E_{0}-E\right)}{b E}-\frac{a}{b}, \quad c E+d F=\gamma
$$

Depending on the parameters, system (2.1) has from one to three stationary points. The case of three stationary points is shown in Figure 2. The points $A$ and $C$ are stable nodes, the point $B$ is a saddle. The point $A$ corresponds to high levels of Fas and low levels of Erk, whereas the point $C$ corresponds to low levels of Fas and high levels of Erk. Hence, the point $A$ is associated with erythroid cell differentiation or apoptosis, the point $C$ with erythroid cell self-renewal.

The basins of attraction of the points $A$ and $C$ are separated by the separatrix of the point $B$. If the initial condition is chosen in a random way on the plane $(E, F)$, then the probability for the trajectory to go to the point $A$ or to the point $C$ depends on the areas of their basins of attraction. Let us denote these probabilities by $p_{A}$ and $p_{C}$, respectively. It can be easily verified that $p_{A}$ increases with the increase of $\gamma$ and decreases with the increase of $\alpha$. Moreover,

$$
p_{A}= \begin{cases}0, & \text { if } \gamma \leq \gamma_{0} \\ 1, & \text { if } \gamma \geq \gamma_{1}\end{cases}
$$

where $\gamma_{0}$ and $\gamma_{1}$ are some given values, $\gamma_{0}<\gamma_{1}$, which depend on $\alpha$. We note that $p_{A}+p_{C}=1$. Hence, if $\gamma$ is sufficiently large the erythroid cell will undergo differentiation or apoptosis, whereas if $\gamma$ is sufficiently small it will self-renew.

Before ending this section, let us briefly concentrate ourselves on the case $\alpha=0$.

When $\alpha=0,(2.1)$ has a stationary point given by $(E, F)=(0, \gamma / d)$. It describes an absence of Erk and a saturation of Fas. Other stationary points are solutions of

$$
F=\frac{\beta}{b} E^{k-1}\left(E_{0}-E\right)-\frac{a}{b} \quad \text { and } \quad c E+d F=\gamma .
$$




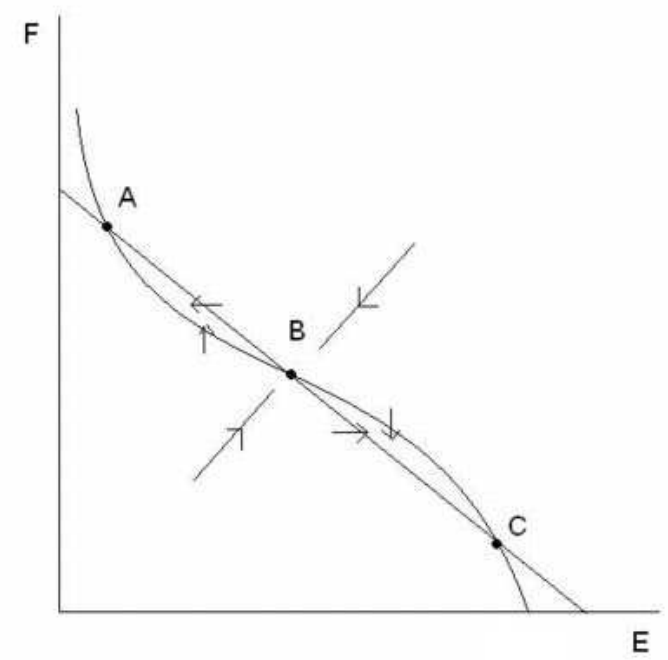

Figure 2: Phase plane of system (2.1).

One can then easily check that (2.2) may have from 0 up to 2 solutions. These stationary solutions have the same behavior as the stationary solutions obtained above for $\alpha>0$, two of them are stable, whereas the third one (located, geometrically, between the other two) is unstable.

Dynamics of the intra-cellular network regulating cell fate (self-renewal, differentiation and death by apoptosis) have been discussed above. The next section is devoted to the nature of cell motion, in particular the use of reaction-diffusion systems for the description of erythroid progenitor dynamics will be dealt with.

\section{Cell division and displacement in individual based modelling}

Spatial cell distribution in the bone marrow should be taken into account in order to specify the conditions of leukemia development. This distribution is closely related to cell division and displacement. In this section we will investigate both cell division and displacement with the individual based modelling approach. We will also use it to explain the origin of random cell motion due to division. This will justify the usage of reaction-diffusion systems in the next section. Simulations will be performed using the software "Cell dynamics", version "Soft sphere model", presented in details in Bessonov et al. [10].

We briefly recall the basis of the individual based model used in the software "Cell dynamics". Let us consider a system of particles on the plane with the coordinates $x_{1}, \ldots, x_{N}$, where $x_{i}$ is a vector with two components. Each particle moves according to the equation

$$
\ddot{x}_{i}+\nu \dot{x}_{i}+\sum_{j \neq i} f\left(r_{i j}\right)=0
$$


where the second term in the left-hand side of this equation describes the friction of the particle by the medium, the last term represents the force acting on the particle by all other particles. This is one of possible models in the approach called dissipative particle dynamics [10]. The force $f\left(r_{i j}\right)$ between the $i$-th and the $j$-th particle depends on the distance $r_{i j}$ between them. In molecular dynamics this is the Van der Waals force, which is nonlocal and usually described by the LennardJones potential. In the case of cell dynamics, the force between two particles is nonzero only when they touch each other, that is when the distance between the centers of the spherical particles is less than the sum of their radii.

Equation (3.1) allows us to determine the position of each cell in time. In addition, the process of cell division, which changes the number of cells and the forces acting between them, must be specified. This is done hereafter.

\subsection{Cell division}

When hematopoietic cells in the bone marrow divide, they push each other resulting in their displacement. This mechanism determines motion of immature red blood cells, which are the most represented cells in the bone marrow. Therefore, in order to study cell motion, we need to begin with cell division.

When a cell divides, it is replaced by two other cells. If we neglect the mechanical interaction with other cells, then the center of mass of the body composed by the two daughter cells does not change in comparison with the center of mass of the mother cell. Hence, after each division, each daughter cell is shifted with respect to its mother cell. Figure 3 shows several first consecutive divisions simulated with the software "Cell dynamics", version "Soft sphere model". Cell size and the frequency of division are given as parameters of the model.
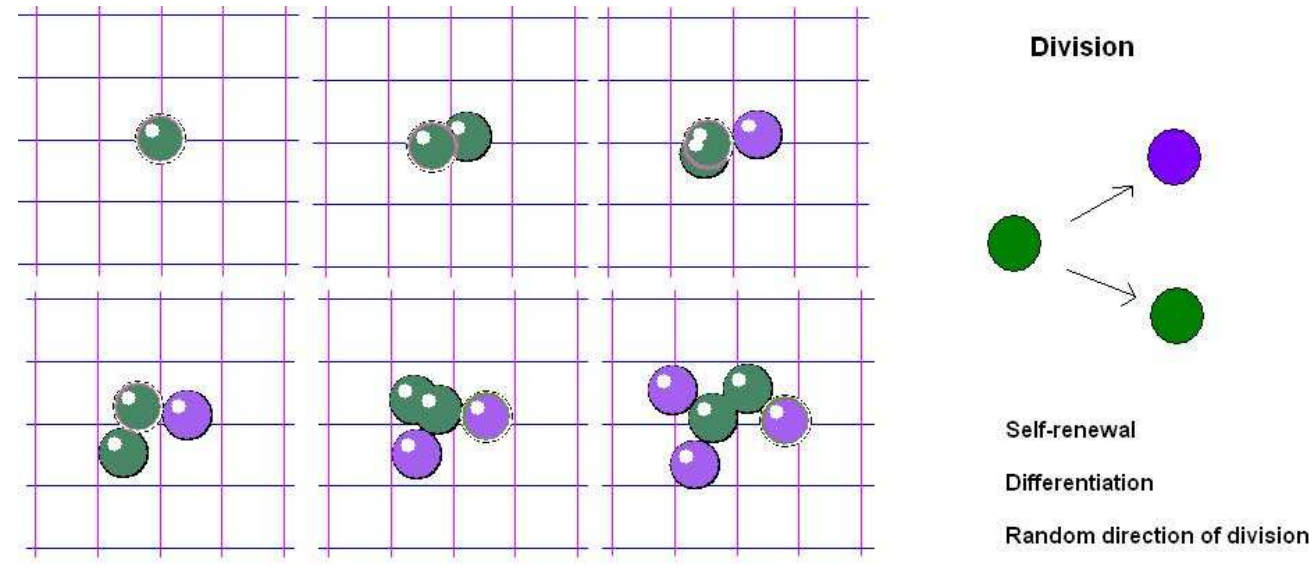

Figure 3: Successive cell divisions simulated with the software "Cell dynamics", version "Soft sphere model". Every division gives birth to two daughter cells (green for a self-renewing division, purple for differentiating division). The direction of division is not specified.

There is one more parameter which should be specified in order to describe cell division: the 
direction of division, that is the direction of the straight line connecting the centers of the new cells. In some cases, the direction of division can be determined by the position of the mother cell with respect to the surrounding cells. In particular, this can be the case in embryogenesis or in plant growth. However, in the case of hematopoiesis, we can assume that this direction is arbitrary. Therefore the angle of the straight line connecting the centers with respect to the $x$-axis is chosen in a random way with a uniform distribution.

Let now focus on cell motion. Consider the following division process $A \rightarrow A+B$ (Figure 3 ) under the assumptions formulated above. After each division the cell $A$ gives one cell, which is identical to itself (self-renewal), and another cell which is different (differentiation). Hence, there will always be one cell of the type $A$ and a growing number of cells $B$. After each division the cell $A$ moves in a random direction at the distance equal to its radius. This means that the cell $A$ moves along the plane in a random way. In the case where there are many such cells and we can consider them as a continuous medium, this motion becomes similar to diffusion and can be described in a similar way. We will discuss this question in more detail in the next section.

Each cell defined in the individual-based model must be able to divide, change its type, or die (either by apoptosis or necrosis). Hence probabilities for such events are provided by the user, for each type of cell defined. These probabilities are determined by intra-cellular regulatory networks. In the case of erythroid progenitors they are discussed in Section 2.

In the example shown in Figure 4 (left), there is one stem cell (green) that divides in two cells at each division, one cell is identical (this is self-renewal) and the other cell is of another type (red). In turn, the red cell can self-renew with the probability 0.57 , differentiate with the probability 0.39 , or die by apoptosis with the probability 0.04 (this probability does not appear on Figure 4, it is obtained from the two other probabilities). In the first case, it divides giving two cells identical to itself. In the second case, it gives two different cells (violet). If the cell dies, it is removed from the computational domain. Red cells can also mutate with the probability 0.001 . When this happens, a mutated cell (yellow) appears. Its properties are different in comparison with the original cell (red). It self-renews with the probability 0.7 , differentiates with the probability 0.3 , and does not die by apoptosis. These assumptions correspond, for instance, to properties of malignant cells. Their self-renewal ability is greater than for normal cells, while differentiation and apoptosis abilities are lower (or absent). Therefore, we can expect that after some time malignant cells will appear and will take over normal cells. It is numerically observed that malignant cells push out normal cells and gradually fill the whole computational domain (Figure 4, right). This replacement corresponds to reaction-diffusion waves discussed in Section 4, Theorem 1. Cell division occurs according to the algorithm described above, presented in Figure 3.

\subsection{Cell displacement}

There are various mechanisms of cell displacement in the bone marrow. We discuss here random cell motion, which is to some extent similar to diffusion, and directed cell motion, similar to convection. Since biological cells are macroscopical objects, the usual Fickian diffusion is not applicable for them. However they can move in a random way due to division.

Let us discuss the motion of a single dividing cell when it is surrounded by other cells. Obvi- 

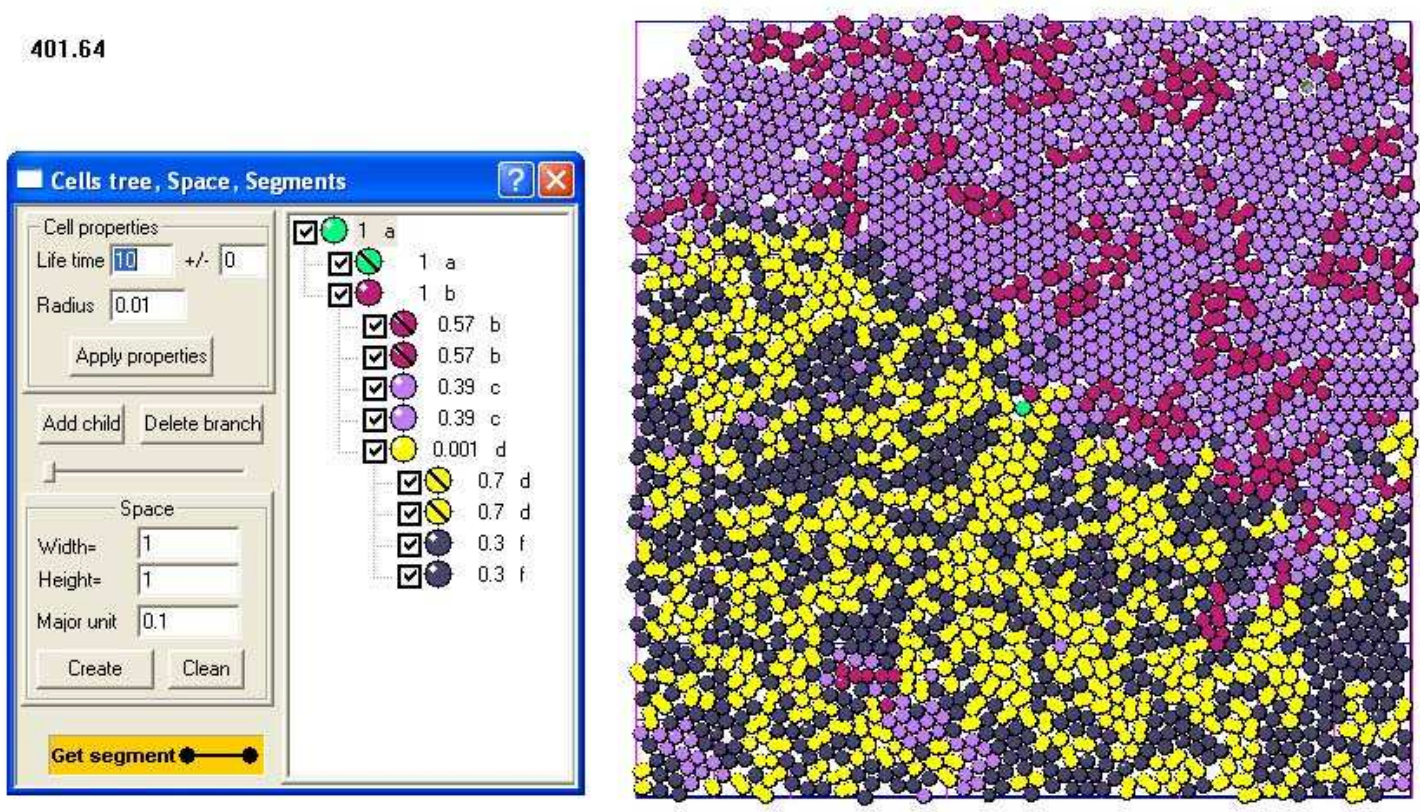

Figure 4: Simulation obtained with the software "Cell dynamics". Left: Properties of the different cells involved in the computations are listed, as self-renewing (same color), differentiating (different color) and mutation (yellow) probabilities. Right: Computational domain, filled with cells defined on the left panel.

ously, cells push each other and create a preferential direction of motion. It is easier for a cell to move in the direction where there are less cells. In order to study this phenomenon, we consider the following numerical experiment. The cell $A$ is placed at the bottom of an empty rectangular box (Figure 5, left). It divides reproducing itself and some other cells which fill the box. These latter cells do not divide and do not move unless they are pushed by other cells. What will be the position of the cell $A$ in time? If it moves in a random way, its distance from the initial position will grow proportionally to $\sqrt{t}$ (with random perturbations). However, when there are many other cells around it, they will influence its motion pushing it to the direction where cell concentration is lower. If we introduce pressure, that is the force exerted by cells on the unit surface, then, similar to Darcy's law, cell velocity will be determined by pressure gradient [13]. It can be verified on model examples that the cell will move in this case with a constant speed, that is its displacement will be proportional to $t$.

We can vary the ratio of the directed and random motion. In order to do this, let us consider the division scheme in the form $A \rightarrow A+B+2 C$ and assume that the cells $A$ and $B$ have the same radius, the radius of the cell $C$ is twice less, the cell $B$ is located on one side of the cell $A$ after the division, and the cells $C$ on the diametrically opposite side. The cell $A$ is shown in yellow in Figure 5, $B$ and $C$ in blue. Clearly, such a cell division is not realistic from the biological point of view. We consider it in order to explain the mechanisms of cell motion. We use here the advantage of mathematical modelling, which allows us to consider idealized models, in order to 


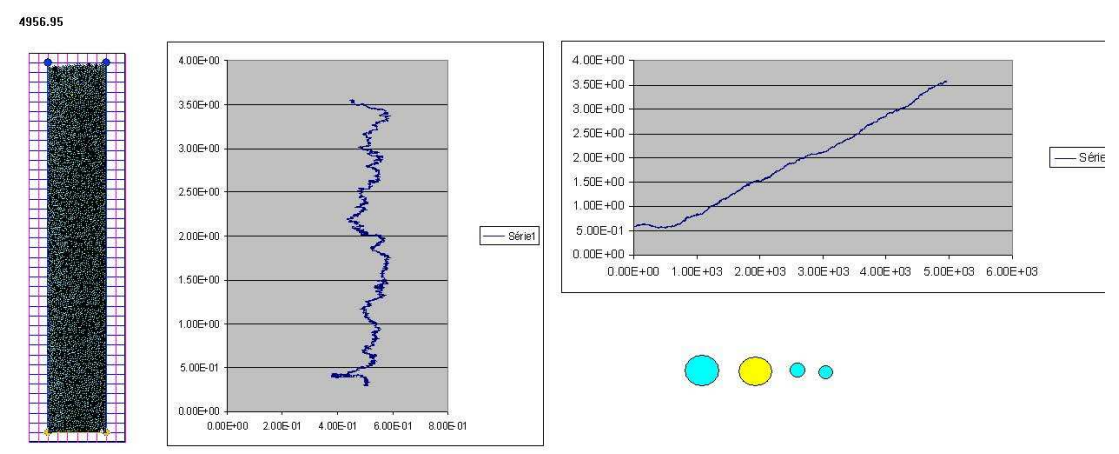

Figure 5: Trajectory and displacement of a cell $A$ dividing according to the process $A \rightarrow A+B+$ $2 C$. Left: Rectangular domain, center: cell trajectory, right: cell displacement.

study a symmetric division where the center of the cell $A$ does not shift after cell division, and various asymmetric divisions where this center displaces due to division. In the real biological situation, division is asymmetric. We will see below that this results in a random cell motion. However if cell density is sufficiently high, convective cell motion becomes also important. To study it more precisely and independently of the random motion, we need to consider a symmetric division.

With the division scheme shown in Figure 5, the center of the cell $A$ before and after the division remains at the same place. In fact, we consider here not the center of mass of the system of cells but its geometrical center. Hence, cell division in this case does not provide random motion. Figure 5 shows the cell trajectory and its displacement from the initial position. We can see that its displacement is close to a linear function. Therefore, it is a directed motion with a weak random component.

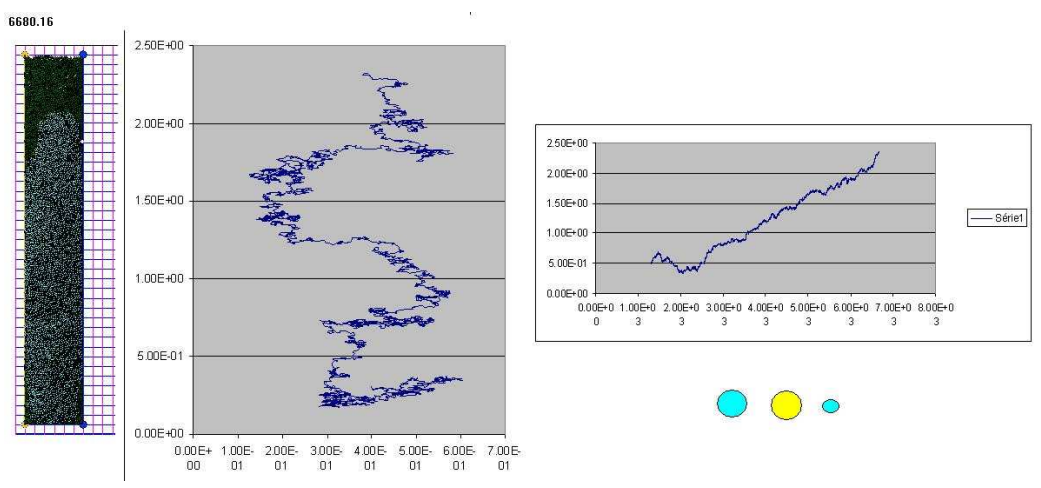

Figure 6: Trajectory and displacement of a cell $A$ dividing according to the process $A \rightarrow A+B+C$, where $B$ and $A$ have the same radius, and the radius of $C$ equals one half of the radius of $A$. Left: Rectangular domain, center: cell trajectory, right: cell displacement. 
Figure 6 shows another scheme of cell division, $A \rightarrow A+B+C$, where $B$ and $C$ are from the opposite sides of $A$. Since the radius $r_{C}$ of $C$ is twice less than the radius $r_{B}$ of $B$, then the center of the cell $A$ moves at $r_{C} / 2$ after each division. The cell displacement remains practically linear. However its trajectory essentially changes in comparison with the previous case. The projection of the cell speed on the vertical direction remains the same since it is determined by the frequency of its division. In other words, by the rate with which it fills the box with other cells. However, its local mobility becomes higher because of the increased random motion. The cell motion has two components: small random displacements and large scale sinusoidal motion from one side to another and upwards. This horizontal motion of the cell is caused by the pressure difference. Since the box is sufficiently large, the cell first fills one side, then another side of the box. Its increased mobility makes it more sensitive to the pressure difference.

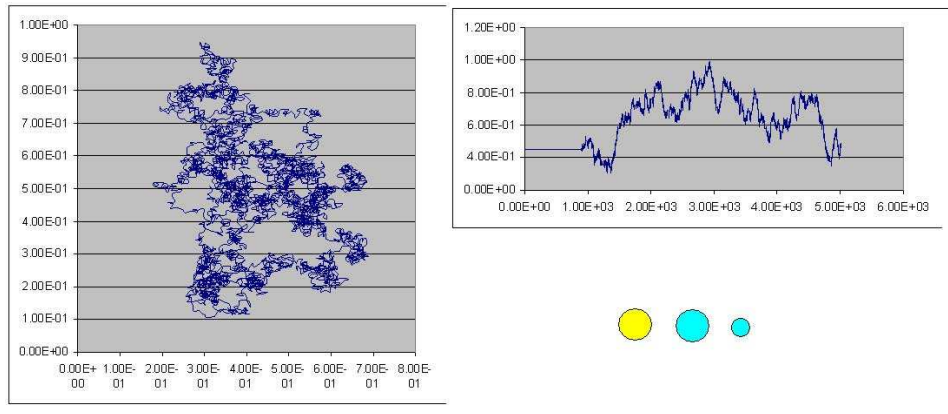

Figure 7: Cell trajectory (left) and displacement (right) in the case of a strong random component.

If we increase even more the random component of the cell motion by appropriately choosing division scheme, then the directed component of its motion is not visible any more (Figure 7). Another parameter which determines the relative contribution of the random and directed components of the cell motion is their concentration. If it is low, then mechanical interaction with the surrounding cells will be negligible.

In the next section we model the evolution of cell populations by reaction-diffusion equations. We take into account their random motion described by the diffusion-terms but not their directed motion. Directed or convective motion should be taken into account if the density of cell population is sufficiently high. In this case it would be described by reaction-diffusion equations coupled with fluid dynamics in a porous medium. Such a model has been studied in previous works $[10,20]$.

We note finally that the diffusion coefficient depends on the cell adhesion to the extracellular matrix. In particular, leukemic cells have a weaker adhesion and, consequently, a bigger diffusion coefficient. 


\section{Approximation of fast intra-cellular regulation}

\subsection{Normal erythropoiesis}

Consider several erythroid progenitor sub-populations denoted by $P_{1}, \ldots, P_{n}$ (see Demin et al. [19] for more details). Each of them consists of identical cells with given rates of self-renewal $s_{i}$, differentiation $d_{i}$ and apoptosis $a_{i}, i=1, \ldots, n$. As discussed in Section 2, these rates and their dependence on the parameters are determined by the Erk/Fas regulatory system (see Figure 1). In the framework of the model described in Section 2, they are determined by the basins of attraction of the corresponding stationary points.

Evolution of these population densities is described by the ordinary differential system of equations

$$
\left\{\begin{array}{l}
\frac{d P_{1}}{d t}=H+\left(s_{1}-d_{1}-a_{1}\right) P_{1}, \\
\frac{d P_{i}}{d t}=\left(s_{i}-d_{i}-a_{i}\right) P_{i}+2 d_{i-1} P_{i-1}, \quad i=2, \ldots, n,
\end{array}\right.
$$

where $H$ denotes a constant flux of hematopoietic stem cells differentiating in erythroid progenitors, the coefficients $s_{i}, d_{i}$ and $a_{i}$ depend on the values of the concentrations of intra-cellular proteins Erk and Fas, $E_{i}$ and $F_{i}$. These concentrations are regulated by intra-cellular networks (see Section 2). In this section we will not take into account the external control feedback by hormones (glucocorticoids or erythropoietin) discussed in Section 2. Hence, the source term $\alpha$ in system (2.1) is assumed to be constant. Fas-ligand, the main external source for Fas activation is produced by mature cells [18], hence we assume $\gamma$, in (2.1), is a function of $P_{j}, j=1, \ldots, n$. Consequently, the protein concentrations are governed by the equations (cf. [19])

$$
\left\{\begin{aligned}
\frac{d E_{i}}{d t} & =\Phi\left(\alpha, E_{i}, F_{i}\right) \\
\frac{d F_{i}}{d t} & =\Psi\left(\sum_{j=1}^{n} \mu_{i j} P_{j}, E_{i}, F_{i}\right)
\end{aligned}\right.
$$

where the functions $\Phi$ and $\Psi$ are defined in (2.1), and $\mu_{i j}$ are constant parameters accounting for the weight of mature cell populations in the production of Fas-ligand.

We now introduce spatial cell distribution. We consider a one-dimensional problem with the space variable $x$ and assume cells can move in space in a random way. This motion can be described by diffusion (see Section 3). The intra-cellular concentrations $E_{i}$ and $F_{i}$ are supposed to be the same for all cells in the $i$-th population, therefore they do not depend on the space variable, but can depend on time.

We also take into account a limitation of cell proliferation when cell density exceeds some threshold level, by means of a chemical cell interaction. Let $P_{0}$ be the maximal cell density. Then system (4.1) can be rewritten in order to take into account both diffusion and the dependence of 
cell proliferation (self-renewal and differentiation) on $P_{0}-\bar{P}$, where $\bar{P}=\sum_{i=1}^{n} P_{i}$,

$$
\begin{aligned}
\frac{\partial P_{1}}{\partial t} & =D \frac{\partial^{2} P_{1}}{\partial x^{2}}+H+\left(s_{1}-d_{1}\right) P_{1}\left(P_{0}-\bar{P}\right)-a_{1} P_{1} \\
\frac{\partial P_{i}}{\partial t} & =D \frac{\partial^{2} P_{i}}{\partial x^{2}}+\left(s_{i}-d_{i}\right) P_{i}\left(P_{0}-\bar{P}\right)-a_{i} P_{i}+2 d_{i-1} P_{i-1}\left(P_{0}-\bar{P}\right), \quad i=2, \ldots, n .
\end{aligned}
$$

In order to be able to analyze the model, we first assume intra-cellular reactions are fast in the time scale related to cell motion. Then, instead of system (4.2), we can consider the stationary equations

$$
\Phi\left(\alpha, E_{i}, F_{i}\right)=0, \quad \Psi\left(\sum_{j=1}^{n} \mu_{i j} P_{j}, E_{i}, F_{i}\right)=0 .
$$

Therefore, $E_{i}$ and $F_{i}$ can be expressed as functions of $P_{j}, j=1, \ldots, n$. As a consequence, coefficients $s_{i}, d_{i}, a_{i}$ in (4.1) also become functions of $P_{j}$.

System (4.3) and (4.4) describes erythroid progenitor dynamics in normal erythropoiesis under the assumption of fast intra-cellular reactions. Consider first the case of a single cell population $P_{1}$. Then system (4.3) is reduced to the first equation where $\bar{P}=P_{1}$ :

$$
\frac{\partial P_{1}}{\partial t}=D \frac{\partial^{2} P_{1}}{\partial x^{2}}+H+\left(s_{1}-d_{1}\right) P_{1}\left(P_{0}-\frac{a_{1}}{s_{1}-d_{1}}-P_{1}\right)
$$

Equation

$$
H+\left(s_{1}-d_{1}\right) P_{1}\left(P_{0}-\frac{a_{1}}{s_{1}-d_{1}}-P_{1}\right)=0
$$

has a unique positive solution $P_{1}=P_{1}^{*}$ if $s_{1}-d_{1}>0$. It is a globally asymptotically stable stationary solution of equation (4.5), that satisfies $P_{1}^{*} \geq \max \left\{0, P_{0}-a_{1} /\left(s_{1}-d_{1}\right)\right\}$.

If $P_{0}>a_{1} /\left(s_{1}-d_{1}\right)$, then $P_{1}^{*} \geq P_{0}-a_{1} /\left(s_{1}-d_{1}\right)$, and $P_{1}^{*}$ is positive even if $H=0$. This means that even in the absence of hematopoietic stem cells, erythroid progenitors keep a positive concentration due to self-sustained proliferation. This case seems to be unrealistic from the biological point of view. Therefore, we will assume in what follows $P_{0} \leq a_{1} /\left(s_{1}-d_{1}\right)$.

In the next section, we introduce a population of leukemic cells and investigate the dynamics of the model. In particular, we focus on the existence of a leukemic equilibrium.

\subsection{Erythroleukemia}

Consider two cell lineages, normal and mutated. The lineage of mutated cells will differ by the rates of self-renewal, differentiation and apoptosis. Denote by $P_{i}$ cells from the normal lineage and by $Q_{i}$ cells from the mutated lineage. Both of them are described by the same model (4.3) as in the previous section. However, we should take here into account that the total number of cells include both lineages,

$$
\bar{P}=P_{1}+\cdots+P_{n}+Q_{1}+\cdots+Q_{n} .
$$

In what follows we restrict ourselves to the case where each of these two lineages consists of a single cell type. This is equivalent to the assumption that we neglect in the previous equality all 
cell densities except for $P_{1}$ and $Q_{1}$. Then equations for $P_{1}$ and $Q_{1}$ become independent of other equations and can be written in the form:

$$
\left\{\begin{array}{l}
\frac{\partial P}{\partial t}=D_{P} \frac{\partial^{2} P}{\partial x^{2}}+H+(s-d) P\left(P_{0}-P-Q\right)-a P \\
\frac{\partial Q}{\partial t}=D_{Q} \frac{\partial^{2} Q}{\partial x^{2}}+\left(s_{m}-d_{m}\right) Q\left(P_{0}-P-Q\right)-a_{m} Q
\end{array}\right.
$$

where the subscripts are omitted for simplicity of notation, and $s_{m}, d_{m}, a_{m}$ denote the rates of self-renewal, differentiation and apoptosis of mutated cells, respectively. In particular, since there is only one generation of erythroid progenitors, denoted by $P$, the influence of progenitor densities on the activation of Fas is neglected, so that (4.4) becomes

$$
\Phi(\alpha, E, F)=0, \quad \Psi(\gamma, E, F)=0,
$$

with $\alpha>0$ and $\gamma>0$ constant. Consequently $E$ and $F$ no longer depend on $P$ and, similarly, the different rates do not depend on cell densities. This assumption has then strong consequences, yet it allows drawing comprehensive conclusions on the existence of a leukemic equilibrium and treatment of the disease (see below).

The constant source $H$ is absent in the second equation of (4.6) because it is assumed in the case of erythroleukemia there is no permanent influx of mutated cells from the stem cell compartment. Different assumptions could of course be considered.

\subsubsection{Existence of a Leukemic Equilibrium}

In order to analyze reaction-diffusion system (4.6), we first consider the system without diffusion,

$$
\left\{\begin{array}{l}
\frac{d P}{d t}=H+(s-d) P\left(P_{0}-P-Q\right)-a P \\
\frac{d Q}{d t}=\left(s_{m}-d_{m}\right) Q\left(P_{0}-P-Q\right)-a_{m} Q
\end{array}\right.
$$

and we assume $s-d>0, s_{m}-d_{m}>0$, and $a_{m} /\left(s_{m}-d_{m}\right)<P_{0}<a /(s-d)$. The assumption $a_{m} /\left(s_{m}-d_{m}\right)<P_{0}$ ensures the possibility to have a leukemic equilibrium (see (4.8)), as shown below.

Zero lines of the right-hand side of (4.7) are given by the equalities

$$
H+k P(-b-P-Q)=0, \quad k_{m} Q\left(b_{m}-P-Q\right)=0,
$$

where

$$
k=s-d, \quad k_{m}=s_{m}-d_{m}, \quad b=-P_{0}+\frac{a}{k}, \quad b_{m}=P_{0}-\frac{a_{m}}{k_{m}} .
$$

According to the above assumptions, all parameters, $k, k_{m}, b$ and $b_{m}$ are positive.

From the first equation in (4.8),

$$
Q=-b-P+\frac{H}{k P}
$$


and from the second equation

$$
Q=0 \quad \text { or } \quad Q=b_{m}-P \text {. }
$$

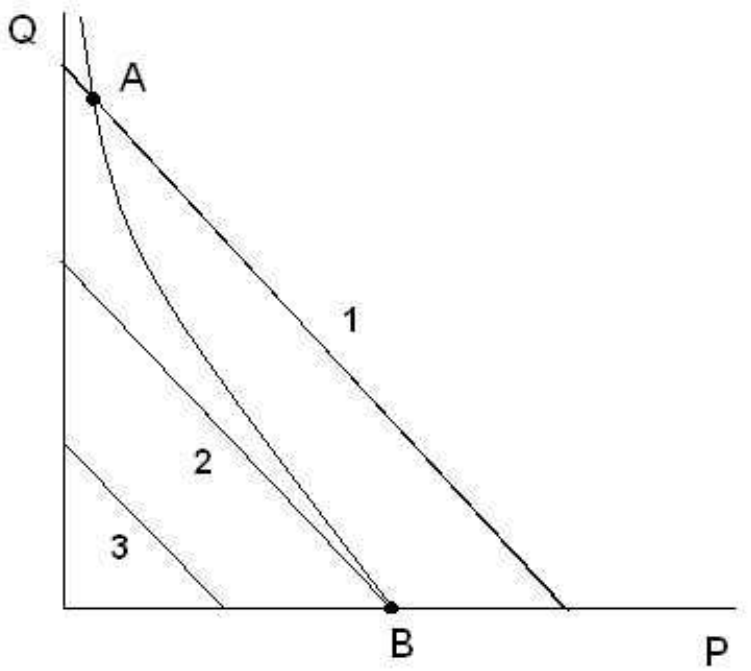

Figure 8: Graphical solution of system (4.9)-(4.10). Case 1 corresponds to the existence of two stationary points, $A$ and $B$, the case 3 to the existence of only one stationary point, $B$. In case 2, the points $A$ and $B$ have the same location.

System (4.9)-(4.10) can have one or two stationary points (Figure 8). If we put $Q=0$ in (4.9), and denote by $P^{*}$ the positive solution of this equation, then we find

$$
P^{*}=\frac{b}{2}\left(\sqrt{1+\frac{4 H}{k b^{2}}}-1\right) .
$$

The stationary solution $(P, Q)=\left(P^{*}, 0\right)$ always exists.

On the other hand, from (4.9) and the second equation in (4.10), we obtain

$$
P_{m}^{*}+Q_{m}^{*}=-b+\frac{H}{k P_{m}^{*}} \quad \text { and } \quad P_{m}^{*}+Q_{m}^{*}=b_{m} .
$$

If $b_{m}>P^{*}$, then we have the case 1 in Figure 8 . There exist two stationary points: $A$ with the coordinates $\left(P_{m}^{*}, Q_{m}^{*}\right)$, and $B$ with the coordinates $\left(P^{*}, 0\right)$. If $b_{m}<P^{*}$, then we have the case 3 in Figure 8 , there exists a unique stationary point $B=\left(P^{*}, 0\right)$. It follows that leukemia may develop only if $b_{m}>P^{*}$.

Condition $b_{m}>P^{*}$ can be written as

$$
b_{m}\left(b_{m}+b\right)>\frac{H}{k}
$$


or equivalently

$$
\left(\mu-\frac{P_{0} k}{a}\right)(\mu-1)>\frac{H k}{a^{2}}
$$

where

$$
\mu=\frac{a_{m}}{a} \frac{k}{k_{m}}=\frac{a_{m}}{a} \frac{s-d}{s_{m}-d_{m}} .
$$

We call the inverse parameter $1 / \mu$ the strength of mutation. If a malignant mutation decreases the rate of apoptosis and differentiation and increases the rate of proliferation, then $\mu<1$. This means that the strength of mutation is greater than 1 .

We recall that the assumption $b>0$ implies that $P_{0} k / a<1$. Moreover, from (4.12) and the assumption $b_{m}>0$, one deduces $\mu<P_{0} k / a$. Consequently, the left-hand side of (4.11) is positive. Furthermore, it is straightforward that inequality (4.11) is satisfied if and only if

$$
P_{0}>\frac{H}{a} \text {. }
$$

This condition means that (4.11) is true for $\mu=0$. Indeed, the set of values of $\mu$ satisfying (4.11) is an interval in the form $\left[0, \mu^{*}\right)$ with $\mu^{*}<P_{0} k / a<1$. If (4.13) is not satisfied, then leukemia will not develop, independently of the strength of mutation. This occurs if the influx of normal cells from the stem cell compartment is sufficiently large.

If (4.11) is satisfied, that is the mutation is sufficiently strong and the influx is not strong enough, then system (4.7) has two stationary points $A$ and $B$ (Figure 8). It can be verified that $A$ is stable while $B$ is unstable. This means that the disease will develop. The values of the concentrations $P_{m}^{*}$ and $Q_{m}^{*}$ in the leukemic equilibrium depend on $\mu$. In particular, the concentration $Q_{m}^{*}$ of malignant cells can be rather low if $\mu$ is close to the critical value. If (4.11) is not satisfied (weak mutation or strong influx), then there exists a unique stable stationary point $B$, which corresponds to the disease free situation.

Before turning to the treatment investigation, let us go back to the reaction-diffusion system (4.6). The analysis of the ordinary differential system of equations (4.7) allows us to do some conclusions about the behavior of solutions of (4.6). More precisely, classical results on monotone systems allow to conclude to the existence of a travelling wave providing a transition from the unstable disease free equilibrium $B$ to the leukemic equilibrium $A$, when (4.11) is satisfied. The existence and stability of travelling waves for monostable monotone reaction-diffusion systems are well known [41]. The existence result is stated in the next theorem.

Theorem 1. Let condition (4.11) be satisfied. Then the endemic equilibrium $\left(P_{m}^{*}, Q_{m}^{*}\right)$ of system (4.6) considered on the whole axis is globally asymptotically stable in the sense that any solution with the initial condition $\left(P_{0}(x), Q_{0}(x)\right)$, where $P_{0}(x) \geq \epsilon, Q_{0}(x) \geq \epsilon, \epsilon$ is a positive constant, converges uniformly to $(P, Q)=\left(P_{m}^{*}, Q_{m}^{*}\right)$ as $t \rightarrow \infty$. The disease free solution $\left(P^{*}, 0\right)$ is unstable.

Moreover there exists a minimal speed $c_{0}$ such that for all speeds $c \geq c_{0}$ there exist travelling wave solutions of system (4.6), that is solutions of the form $P(x, t)=p(x-c t), Q(x, t)=q(x-c t)$. The functions $p(x)$ and $q(x)$ are monotone with respect to $x$ and satisfy the system

$$
D_{P} p^{\prime \prime}+c p^{\prime}+H+(s-d) P\left(P_{0}-P-Q\right)-a P=0,
$$




$$
D_{Q} q^{\prime \prime}+c q^{\prime}+\left(s_{m}-d_{m}\right) Q\left(P_{0}-P-Q\right)-a_{m} Q=0
$$

and the conditions at infinity:

$$
p(-\infty)=P_{m}^{*}, \quad q(-\infty)=Q_{m}^{*}, \quad p(+\infty)=P^{*}, \quad q(+\infty)=0 .
$$

For $c<c_{0}$ such solutions do not exist.

The proof of the theorem is based on the reduction of system (4.6) to a monotone system and on the application of the comparison principle. The interested reader may refer to [41] for details.

It is known that the appearance of malignant cells is due to several rare consecutive mutations. Once this event occurs, malignant cells start proliferating. If malignant cells appear then they persist and spread in the whole bone marrow in the form of a travelling wave. Their existence is stated in Theorem 1.

In the following, we investigate potential treatment of leukemia that is, from a modeling point of view, how can we make the leukemic equilibrium disappear?

\subsubsection{Treatment}

Treatment of acute myeloid leukemia by chemotherapy, in particular by Ara-C (cytosine arabinoside), decreases cell proliferation and increases apoptosis. In the simplest case, in order to model treatment of erythroleukemia, we can assume proliferation and apoptosis rates of normal and malignant cells are proportional to some parameter $\tau$ characterizing the intensity of treatment:

$$
s^{\tau}=s / \tau, \quad d^{\tau}=d / \tau, \quad a_{\tau}=\tau a, \quad s_{m}^{\tau}=s_{m} / \tau, \quad d_{m}^{\tau}=d_{m} / \tau, \quad a_{m}^{\tau}=\tau a_{m},
$$

and $H^{\tau}=H / \tau$. Here $\tau \geq 1$, so in the absence of treatment the coefficients are not modified. Moreover, an increase of $\tau$ increases apoptosis rates while it decreases self-renewal and differentiation rates, for both normal and leukemic cells, and it decreases the stem cell influx. Under these assumptions, the parameter $\mu$ defined in (4.12) does not depend on $\tau$. Treatment will be successful if for a given $\mu$ there exists $\tau$ such that

$$
\left(\mu-\frac{P_{0} k}{a \tau^{2}}\right)(\mu-1) \leq \frac{H k}{a^{2} \tau^{4}}
$$

In particular, we can take $\tau=\sqrt{P_{0} k /(a \mu)}$ for which the left-hand side of this inequality vanishes. Consequently, there exists $\tau>1$ such that (4.14) holds true. In fact, we can be more accurate.

Define the function

$$
f(\tau)=\frac{H k}{a^{2} \tau^{4}}-\left(\mu-\frac{P_{0} k}{a \tau^{2}}\right)(\mu-1), \quad \tau \geq 1,
$$

and suppose $f(1)<0$, that is the leukemic equilibrium exists without treatment ((4.11) holds true). Then one easily obtains

$$
f^{\prime}(\tau)=2 \frac{k}{a \tau^{3}}\left[(1-\mu) P_{0}-2 \frac{H}{a \tau^{2}}\right] .
$$


Since $\mu<1$, then $f^{\prime}(\tau)>0$ if and only if

$$
\tau>\sqrt{2 \frac{H}{a(1-\mu) P_{0}}}:=\tilde{\tau} .
$$

One can note that either $\tilde{\tau} \leq 1$ or $\tilde{\tau}>1$, depending on the parameters. Consequently, if $\tilde{\tau} \leq 1$, the function $f$ is strictly increasing. It crosses the $x$-axis for a given $\tau$ and

$$
\lim _{\tau \rightarrow \infty} f(\tau)=\mu(1-\mu)>0
$$

For a sufficiently large drug dose, the treatment is then successful. If $\tilde{\tau}>1$, then the function $f$ first decreases from $f(1)<0$, until $\tau=\tilde{\tau}$, then $f$ increases, crosses the x-axis and reaches its upper limit (mentioned above). Hence, the treatment will also be successful, yet for $1 \leq \tau \leq \tilde{\tau}$ the treatment worsens the disease (in fact, even for $\tau$ larger than $\tilde{\tau}$, but not too large).

If we consider $H^{\tau}$ in the form

$$
H^{\tau}=H \frac{\tau-\tau_{1}}{1-\tau_{1}}, \quad \tau_{1}>1,
$$

which takes into account that intensive chemotherapy kills all stem cells $\left(\tau=\tau_{1}\right)$, then instead of (4.14) we have

$$
\left(\mu-\frac{P_{0} k}{a \tau^{2}}\right)(\mu-1) \leq \frac{H k\left(\tau-\tau_{1}\right)}{a^{2} \tau^{3}\left(1-\tau_{1}\right)}, \quad \tau \in\left[1, \tau_{1}\right]
$$

Let define

$$
g(\tau)=\frac{H k\left(\tau-\tau_{1}\right)}{a^{2} \tau^{3}\left(1-\tau_{1}\right)}-\left(\mu-\frac{P_{0} k}{a \tau^{2}}\right)(\mu-1), \quad \tau \in\left[1, \tau_{1}\right],
$$

with $g(1)<0$, to ensure the existence of the leukemic equilibrium in the absence of treatment. Simple computations lead to

$$
g^{\prime}(\tau)=\frac{k}{a \tau^{3}}\left[2(1-\mu) P_{0}+\frac{H}{a\left(1-\tau_{1}\right)}\left(3 \frac{\tau_{1}}{\tau}-2\right)\right] .
$$

One can note that, since $\tau \leq \tau_{1}$ then $3 \tau_{1} / \tau-2>0$, and $g^{\prime}(\tau)>0$ if and only if

$$
\tau>\frac{3 \tau_{1}}{2\left[\frac{H}{a}(1-\mu)\left(\tau_{1}-1\right) P_{0}+1\right]}:=\bar{\tau} .
$$

Hence, similarly to what we previously concluded for function $f$, the function $g$ can either be increasing (if $\bar{\tau} \leq 1$ ), or first decreases from $g(1)<0$, until it reaches $\bar{\tau}$, then provided that $\bar{\tau}<\tau_{1}$ it increases up to $g\left(\tau_{1}\right)$. We focus on the second case. One can note that

$$
g\left(\tau_{1}\right)=-\left(\mu-\frac{P_{0} k}{a \tau_{1}^{2}}\right)(\mu-1)
$$

and can be either negative if $\mu \tau_{1}^{2}<P_{0} k / a$, or positive if $\mu \tau_{1}^{2}>P_{0} k / a$. Thus, various cases can occur: First, if $\bar{\tau}>\tau_{1}$ then the function $g$ is decreasing on the interval $\left[1, \tau_{1}\right]$ and treatment only worsens the disease. Second, if $\bar{\tau}<\tau_{1}$ and $g\left(\tau_{1}\right)<0$, then the treatment can worsen the disease, and whatever the administered drug dose the treatment can not cure the disease. Finally, if $\bar{\tau}<\tau_{1}$ and $g\left(\tau_{1}\right)>0$ then treatment can worsen the disease (small doses), but eventually the treatment can cure the disease. This last situation is similar to the above situation, obtained when $H^{\tau}=H / \tau$. 


\section{Discussion}

The aim of this work was twofold. First, we derived and justified mathematical models of erythropoiesis. Often models of cell populations are semi-empiric and based on some intuitive assumptions, which seem in agreement with biological observations. We attempted to give here some more rigorous justification of one of such assumptions about cell displacement. Although there are different mechanisms of cell motion in the bone marrow, we did not discuss here biologically active mechanisms such as chemotaxis but concentrated only on pure mechanical cell interaction. Since new cells appear due to proliferation, cells push each other creating random motion, which can be described by diffusion, and directed motion determined by pressure difference. We carried out some analysis of this motion with individual based modelling. Though the results seem to be convincing, much should be done yet in this direction to have a sufficiently precise description of cell motion in terms of continuum mechanics.

Another important point of the model is related to intra-cellular regulatory networks which determine cell behavior. In the case of erythroid progenitors studied in this work we specified the probabilities of their self-renewal, differentiation and apoptosis. Their values are crucial from the point of view of the evolution of cell population. We used them in the reaction-diffusion model and in cell dynamics modelling.

The second aim of this work was related to the analysis of these models in normal and pathological cases. In particular, if self-renewal is greater than it should be while differentiation and apoptosis are less, hemostasis can be unbalanced resulting in malfunctioning of the whole hematopoietic system. This can be related to malignant mutations. We introduced the parameter, which we called the strength of mutation, which shows whether leukemia will develop or not. In the case of strong mutations, a second, leukemic equilibrium appears. The normal equilibrium still exists but it becomes unstable.

We modelled treatment of leukemia by chemotherapy, considering its action on the rates of proliferation and apoptosis. In the case of acute myeloid leukemia, chemotherapy kills proliferating cells, both normal and leukemic. If it kills all leukemic cells, then it should also kill all normal cells. If some leukemic cells are not killed, this can result in relapse. Why then can treatment be successful and what can cause its failure?

The development of leukemia is determined by the competition of normal and malignant cells for space. Malignant cells are more aggressive in the sense that they proliferate more and die less. The disadvantage of normal cells can be compensated by their influx from the stem cell compartment. The success or failure of treatment in the framework of our model depends on the action of chemotherapy on normal stem cells. The analysis of the model showed that different situations are possible. If normal stem cells are not strongly influenced, then treatment can be successful. This is in agreement with medical observations that normal stem cells are more resistant to Ara-C, the main chemotherapeutic agent. On the other hand, insufficient treatment can promote leukemic cells and worsen the situation. This can happen because treatment kills cells and liberates place for new cells. Since leukemic cells proliferate more, they can then gain space. If normal stem cells are strongly influenced by chemotherapy, it is possible that treatment fails whatever the drug dose. 
We are aware that the model considered in this work is incomplete. We did not take into account deliberately some of the factors such as the influence of certain hormones on erythroid progenitors (erythropoietin, glucocorticoids), pharmaco-kinetics of chemotherapy, resistance to chemotherapy and so on. Moreover, although we presented a rather complex model of erythroid progenitor dynamics (a system of reaction-diffusion equations coupled to a system of nonlinear ordinary differential equations describing regulatory intracellular protein network), we analyzed a simplified version of the model, in order to be able to obtain clear and understandable results. We began this study with the most simple model in order to reveal its basic properties. More complete models will be studied in subsequent works, including in particular confrontation to experimental data for leukemia treatment.

\section{Acknowledgements}

The authors are grateful to S. Bernard, O. Gandrillon, S. Génieys and L. Pujo-Menjouet for useful discussions.

\section{References}

[1] M. Adimy, and F. Crauste, Modelling and asymptotic stability of a growth factor-dependent stem cells dynamics model with distributed delay, Discrete Cont. Dyn. Syst. Ser. B 8 (2007), pp. 19-38.

[2] M. Adimy, F. Crauste, and A. El Abdllaoui, Asymptotic Behavior of a Discrete Maturity Structured System of Hematopoietic Stem Cells Dynamics with Several Delays, Math. Model. Nat. Phenom., 1 (2006), No. 2, pp. 1-22.

[3] M. Adimy, F. Crauste, and S. Ruan, A mathematical study of the hematopoiesis process with applications to chronic myelogenous leukemia, SIAM J. Appl. Math., 65 (2005), pp. 13281352.

[4] M. Adimy, F. Crauste, and S. Ruan, Periodic Oscillations in Leukopoiesis Models with Two Delays, J. Theor. Biol., 242 (2006), pp. 288-299.

[5] M. Adimy, F. Crauste, and S. Ruan, Modelling hematopoiesis mediated by growth factors with applications to periodic hematological diseases, Bull. Math. Biol., 68 (2006), pp. 23212351.

[6] A.R.A. Anderson, K.A. Rejniak, P. Gerlee, and V. Quaranta, Modelling of Cancer Growth, Evolution and Invasion: Bridging Scales and Models, Math. Model. Nat. Phenom. 2 (2007), pp.1-27. 
[7] A. Bauer, F. Tronche, O. Wessely, C. Kellendonk, H.M. Reichardt, P. Steinlein, G. Schutz, and H. Beug, The glucocorticoid receptor is required for stress erythropoiesis, Genes Dev. 13 (1999), pp. 2996-3002.

[8] J. Bélair, M.C. Mackey, and J.M. Mahaffy, Age-structured and two delay models for erythropoiesis, Math. Biosci. 128 (1995), pp. 317-346.

[9] S. Bernard, J. Bélair, and M.C. Mackey, Oscillations in cyclical neutropenia: new evidence based on mathematical modeling, J. Theor. Biol. 223 (2003), pp. 283-298.

[10] N. Bessonov, L. Pujo-Menjouet, and V. Volpert. Cell modelling of hematopoiesis. Math. Model. Nat. Phenom., 1 (2006), No. 2, pp. 81-103.

[11] N. Bessonov, I. Demin, L. Pujo-Menjouet, and V. Volpert. A multi-agent model describing self-renewal or differentiation effect of blood cell population. Mathematical and computer modelling, 49 (2009), pp. 2116-2127.

[12] D. Bonnet, Haematopoietic stem cells, Pathol. 197 (2002), pp. 430-440.

[13] V. Capasso, and D. Bakstein. An introduction to continuous-time stochastic processes. Birkhauser, Boston, 2005.

[14] C. Colijn, and M.C. Mackey, A mathematical model of hematopoiesis-I. Periodic chronic myelogenous leukemia, J. Theor. Biol. 237 (2005), pp. 117-132.

[15] C. Colijn, and M.C. Mackey, A mathematical model of hematopoiesis-II. Cyclical neutropenia, J. Theor. Biol. 237 (2005), pp. 133-146.

[16] F. Crauste, L. Pujo-Menjouet, S. Génieys, C. Molina, and O. Gandrillon, Adding self-renewal in committed erythroid progenitors improves the biological relevance of a mathematical model of erythropoiesis, J. Theor. Biol. 250 (2008), pp. 322-338.

[17] S. Dazy, F. Damiola, N. Parisey, H. Beug, and O. Gandrillon, The MEK-1/ ERKs signalling pathway is differentially involved in the self-renewal of early and late avian erythroid progenitor cells, Oncogene, 22 (2003), pp. 9205-9216.

[18] R. De Maria, U. Testa, L. Luchetti, A. Zeuner, G. Stassi, E. Pelosi, R. Riccioni, N. Felli, P. Samoggia, and C. Peschle, Apoptotic role of Fas/Fas ligand system in the regulation of erythropoiesis, Blood, 93.3 (1999), pp. 796-803.

[19] I. Demin, F. Crauste, O. Gandrillon, and V. Volpert, A multi-scale model of erythropoiesis, Journal of Biological Dynamics (in press). DOI: 10.180/17513750902777642

[20] A. Ducrot, and V. Volpert. On a model of leukemia development with a spatial cell distribution. Math. Model. Nat. Phenom., 2 (2007), No. 3, 101-120. 
[21] O. Gandrillon, U. Schmidt, H. Beug, and J. Samarut, TGF-beta cooperates with TGF-alpha to induce the self-renewal of normal erythrocytic progenitors: evidence for an autocrine mechanism, EMBO J. 18 (1999), pp. 2764-2781.

[22] S. Huang, Y.-P.Guo, G. May, and T. Enver, Bifurcation dynamics in lineage-commitment in bipotent progenitor cells, Dev. biol. 305 (2007), pp. 695-713.

[23] M.J. Koury, and M.C. Bondurant, Erythropoietin retards DNA breakdown and prevents programmed death in erythroid progenitor cells, Science, 248 (1990), pp. 378-381.

[24] C. Lacombe, and P. Mayeux, Biology of erythropoietin, Haematologica, 83 (1998), pp. 724 732.

[25] M. von Lindern, W. Zauner, G. Mellitzer, P. Steinlein, G. Fritsch, K. Huber, B. Lwenberg, and $\mathrm{H}$. Beug, The glucocorticoid receptor cooperates with the erythropoietin receptor and c-Kit to enhance and sustain proliferation of erythroid progenitors in vitro, Blood, 94 (1999), pp. 550-559.

[26] M.C. Mackey, Unified hypothesis of the origin of aplastic anaemia and periodic hematopoiesis, Blood, 51 (1978), pp. 941-956.

[27] F.M. Mazzella, C. Alvares, A. Kowal-Vern, and H.R. Schumacher, The acute erythroleukemias, Clin. Lab. Med., 20 (2000), pp. 119-37.

[28] V. Munugalavadla, and R. Kapur, Role of c-Kit and erythropoietin receptor in erythropoiesis, Critical Reviews in Oncology/Hematology, 54 (2005), pp. 63-75.

[29] J.D. Murray, Mathematical Biology, Springer, New York, 2004.

[30] B. Pain, C.M. Woods, J. Saez, T. Flickinger, M. Raines, S. Peyrol, C. Moscovici, M.G. Moscovici, H.J. Kung, P. Jurdic, et al., EGF-R as a hemopoietic growth factor receptor: the c-erbB product is present in normal chicken erythrocytic progenitor cells and controls their self-renewal, Cell, 65 (1991), pp. 37-46.

[31] J.C. Panetta, W.E. Evans, and M.H. Cheok, Mechanistic mathematical modeling of mercaptopurine effects on cell cycle of human acute lymphoblastic leukemia cells, Br. Journal of Cancer, 94 (2006), pp. 93-100.

[32] L. Pujo-Menjouet, and M.C. Mackey, Contribution to the study of periodic chronic myelogenous leukemia, C.R. Biol. 327 (2004), pp. 235-244.

[33] I. Roeder, and I. Glauche, Towards an understanding of lineage specification in hematopoietic stem cells: A mathematical model for the interaction of transcription factors GATA-1 and PU.1, J. Theor. Biol. 241 (2006), pp. 852-865. 
[34] C. Rubiolo, D. Piazzolla, K. Meissl, H. Beug, J.C. Huber, A. Kolbus, and M. Baccarini, A balance between Raf-1 and Fas expression sets the place of erythroid differentiation, Blood, 108 (2006), pp. 152-159.

[35] J.E. Rubnitz, B. Gibson, and B.O. Smith, Acute myeloid leukemia, Pediatr. Clin. North. Am., 55 (2008), 1, pp. 21-51.

[36] E. Shochat, S.M. Stemmer, and L. Segel, Human haematopoiesis in steady state and following intense perturbations, Bull. Math. Biol., 64 (2002), pp. 861-886.

[37] U. Testa, Apoptotic mechanisms in the control of erythropoiesis, Leukemia, 18 (2004), pp. $1176-1199$.

[38] W. Vainchenker, A. Dusa, and S.N. Constantinescu, JAKs in pathologies: Role of Janus kinases in hematopoietic malignancies and immunodeficiencies, Seminars in Cell and Developmental Biology, 19 (2008), pp. 385-393.

[39] V. Vainstein, Y. Ginosar, M. Shoham, D.O. Ranmar, A. Ianovski, and Z. Agur, The complex effect of granulocyte colony-stimulating factor on human granulopoiesis analyzed by a new physiologically-based mathematical model, J. Theor. Biol., 234 (2005), pp. 311-327.

[40] V. Vainstein, Y. Ginosar, M. Shoham, A. Ianovski, A. Rabinovich, Y. Kogan, V. Selitser, and Z. Agur, Improving Cancer Therapy by Doxorubicin and Granulocyte Colony-Stimulating Factor: Insights from a Computerized Model of Human Granulopoiesis, Math. Model. Nat. Phenom., Vol. 1, No. 2 (2006), pp. 70-80.

[41] A. Volpert, Vl. Volpert, Vit. Volpert. Travelling wave solutions of parabolic systems. AMS, Providence, 1994.

[42] F.M. Watt, and B.L. Hogan, Out of Eden: stem cells and their niches, Science, 287 (2000), pp. 1427-1430.

[43] I.L. Weissman, Stem cells: units of development, units of regeneration, and units in evolution, Cell 100 (2000), pp. 157-168. 